\title{
Диэлектрические свойства нанокристаллического оксида вольфрама в области температур 223-293K
}

\author{
(C) С.А. Козюхин ${ }^{1,3}$, С.А. Бедин ${ }^{2}$, П.Г. Рудаковская ${ }^{1}$, О.С. Иванова ${ }^{1}$, В.К. Иванов ${ }^{1,4}$ \\ ${ }^{1}$ Институт общей и неорганической химии им. Н.С. Курнакова Российской академии наук, \\ 119991 Москва, Россия \\ ${ }^{2}$ Московский педагогический государственный университет, \\ 119991 Москва, Россия \\ ${ }^{3}$ Национальный исследовательский Томский государственный университет (химический фракультет), \\ 634050 Томск, Россия \\ ${ }^{4}$ Московский технологический университет, \\ 119454 Москва, Россия \\ E-mail: sergkoz@igic.ras.ru
}

(Получена 30 августа 2017 г. Принята к печати 12 сентября 2017 г.)

Исследованы диэлектрические свойства нанокристаллического оксида вольфрама в интервале температур $223-293 \mathrm{~K}$ и в диапазоне частот $v=10^{-2}-10^{6}$ Гц. Порошки $\mathrm{WO}_{3}$ с размерами частиц 110,150 и $200 \mathrm{Hм}$ были приготовлены термической обработкой паравольфрамата аммония при различных температурах. Установлено, что для всех образцов частотные зависимости проводимости являются возрастающими с увеличением частоты, в то время как поляризационные характеристики $\varepsilon^{\prime}(v)$ и $\varepsilon^{\prime \prime}(v)$ уменьшаются. Найдено, что частотные зависимости проводимости описываются функцией вида $v^{s}$ с показателем степени, находящимся в диапазоне $(0.83-0.90) \pm 0.01$, что свойственно „прыжковому“ механизму перемещения заряженных частиц (комплексов) по локализованным состояниям, ограниченным потенциальными барьерами и дефектами структуры.

DOI: 10.21883/FTP.2018.07.46046.8719

\section{1. Введение}

Оксид вольфрама $\left(\mathrm{WO}_{3}\right)$ является широкозонным полупроводником $n$-типа проводимости с энергией запрещенной зоны, лежащей в интервале от 2.7 до 3.15 эВ в зависимости от концентрации кислородных вакансий [1]. Он используется в различных областях техники в составе газовых сенсоров [2,3], а также в электрохромных устройствах [4]. В основе применения $\mathrm{WO}_{3}$ лежат электрохромные, а также термохромные свойства данного материала, хорошие оптические модуляционные и электрические характеристики по сравнению с другими оксидами переходных металлов. Круг потенциальных применений $\mathrm{WO}_{3}$ достаточно широк - это „умные окна“, автомобильные стекла, солнечная энергетика, антиотражающие покрытия [5-10].

Обладая ярко выраженной анизотропией кристаллической решетки, оксиды вольфрама способны образовывать ряд полиморфных модификаций от $\alpha$ - до $\varepsilon$-фазы в зависимости от состава, температуры и давления. Каждая из этих фаз отличается специфическим набором физико-химических характеристик. Так, в зависимости от содержания кислорода оксиды вольфрама являются диэлектриками, полупроводниками или даже сверхпроводниками $[11,12]$; в области отрицательных температур ниже $-40^{\circ} \mathrm{C}$ также существует стабильная ферроэлектрическая фаза $\varepsilon-\mathrm{WO}_{3}[13-15]$.

Диэлектрические свойства оксида вольфрама исследовались в нескольких работах [13,16-18], при этом изучались как тонкие пленки в разном фазовом состоянии (поликристаллические [16], аморфные [18]), так и керамические образцы, спрессованные из порошка $\mathrm{WO}_{3}$ и затем отожженные [13,17]. Данные исследования позволили выявить характер изменения таких характеристик, как диэлектрическая проницаемость, диэлектрические потери и тангенс угла диэлектрических потерь, проводимость на переменном токе и соответственно энергия активации проводимости в широком интервале частот и температур. Анализ результатов, полученных в вышеуказанных работах, показал, что фазовое состояние и способ приготовления образцов оказывают самое существенное влияние не только на абсолютные значения диэлектрических параметров, но даже на само их поведение. Например, авторы работы [16] установили, что диэлектрическая проницаемость $\varepsilon^{\prime}$ с ростом температуры уменьшается для тонких пленок $\mathrm{WO}_{3}$, а в работе [17] тот же параметр с ростом температуры увеличивается для массивного оксида вольфрама, при этом абсолютные значения также сильно различаются, соответственно 350 (при угловой частоте $\omega=1$ кГц) и $1.2 \cdot 10^{5}(\omega=2$ кГц $)$. Влияние физико-химических параметров образцов $\mathrm{WO}_{3}$ на диэлектрические свойства является малоизученным. Необходимо также отметить, что все опубликованные исследования выполнялись при температурах выше комнатной, а в области отрицательных температур измерения не проводились, хотя они позволяют получить новые данные о поляризационных процессах в материалах.

Цель данного исследования состояла в изучении диэлектрических характеристик нанокристаллического оксида вольфрама в температурном диапазоне от $T=223$ до $293 \mathrm{~K}$ и выявлении влияния размеров наночастиц на диэлектрические свойства. 
Таблица 1. Результаты РФА и низкотемпературной адсорбции азота для образцов нанокристаллического оксида вольфрама

\begin{tabular}{c|c|c|c|c|c|c|c|c}
\hline \multirow{2}{*}{ Образец } & \multirow{2}{*}{$\begin{array}{c}\text { Удельная площадь } \\
\text { поверхности } \\
\text { по БЭТ, } \mathrm{M}^{2} / \Gamma\end{array}$} & $\begin{array}{c}\text { Размер } \\
\text { ОКР, } \\
\text { нм }\end{array}$ & $\begin{array}{c}\text { Размер } \\
\text { частиц, } \\
\text { нм }\end{array}$ & $\begin{array}{c}\text { Величина } \\
\text { микро- } \\
\text { напряжений }\end{array}$ & \multicolumn{4}{|c}{ Парамеры элементарной ячейки } \\
\cline { 5 - 9 } & 9.3 & 64 & 110 & 0.086 & $\begin{array}{c}7.31279 \\
(55)\end{array}$ & $\begin{array}{c}7.53870 \\
(53)\end{array}$ & $\begin{array}{c}7.70021 \\
(59)\end{array}$ & $\begin{array}{c}90.3174 \\
(60)\end{array}$ \\
\hline $\mathrm{WO}_{3}(600)$ & 4.9 & 65 & 150 & 0.112 & $\begin{array}{c}7.31279 \\
(55)\end{array}$ & $\begin{array}{c}7.53870 \\
(53)\end{array}$ & $\begin{array}{c}7.70021 \\
(59)\end{array}$ & $\begin{array}{c}90.3174 \\
(60)\end{array}$ \\
\hline $\mathrm{WO}_{3}(700)$ & 1.6 & 68 & 200 & 0.139 & $\begin{array}{c}7.3077 \\
(12)\end{array}$ & $\begin{array}{c}7.5309 \\
(12)\end{array}$ & $\begin{array}{c}7.6963 \\
(12)\end{array}$ & $\begin{array}{c}90.5560 \\
(59)\end{array}$
\end{tabular}

\section{2. Методика эксперимента}

Порошки оксида вольфрама были получены термической обработкой $B$-паравольфрамата аммония $\left(\mathrm{NH}_{4}\right)_{10}\left(\mathrm{H}_{2} \mathrm{~W}_{12} \mathrm{O}_{42}\right) \times 4 \mathrm{H}_{2} \mathrm{O} \quad$ (ос.ч.) при температурах 600, 700 и $800^{\circ} \mathrm{C}$ внесением в предварительно разогретую печь на 10 мин с последующей закалкой на воздухе. После синтеза порошки $\mathrm{WO}_{3}$ смешивали с водой и дезагрегировали в ультразвуковой (УЗ) ванне с последующей сушкой на воздухе. Соответственно было получено три типа порошков нанокристаллического оксида вольфрама: $\mathrm{WO}_{3}(600), \mathrm{WO}_{3}(700)$ и $\mathrm{WO}_{3}(800)$.

Диэлектрические свойства исследовали с помощью диэлектрического релаксометра NOVOCONTROL Alpha-A (Novocontrol Technologies) с активной измерительной ячейкой. Измерения проводились в диапазоне частот $v=10^{-2}-10^{6}$ Гц, в интервале температур от 223 до $293 \mathrm{~K}$ в атмосфере азота. Амплитуда напряжения, подаваемого на образец, составляла $1 \mathrm{~B}$.

Рентгенофазовый анализ (РФА) образцов проводили на дифрактометре Bruker D8 Advance $\left(\mathrm{Cu} K_{\alpha}\right.$-излучение, длина волны $\lambda=0.15481 \mathrm{Hм})$ в диапазоне углов $3-100^{\circ}$ по $2 \theta$, с шагом $0.02^{\circ}$ по $2 \theta$, с вращением кюветы (30 об/мин) при скорости вращения гониометра $2 \%$ мин по 20. Для полнопрофильного анализа дифрактограмм использовали программное обеспечение JANA2006 и TOPAS 4.2. Базовую линию описывали полиномами Чебышева 3-й или 4-й степени, используя дополнительный обратный член. Несимметричность пиков учитывали путем численного моделирования по фундаментальным параметрам геометрии съемки. Значения фактора недостоверности $\left(R_{w p}\right)$ для полученных описаний экспериментальных дифрактограмм находились в диапазоне от 2 до 4.

Величины удельной площади поверхности определяли методом низкотемпературной адсорбции азота с помощью анализатора Катакон АТX 06 с использованием модели Брюнауэра-Эммета-Теллера (БЭТ) по 5 точкам в диапазоне парциальных давлений азота 0.05-0.25. Перед измерениями проводили дегазацию образцов в токе сухого гелия при $100^{\circ} \mathrm{C}$ в течение 30 мин. Плотности образцов были рассчитаны по отношению их массы к объему.
Рентгеноспектральный микроанализ осуществляли с использованием растрового электронного микроскопа Carl Zeiss NVision 40, оснащенного детектором Oxford Instruments X-MAX, при ускоряющем напряжении 20 кВ.

\section{3. Экспериментальные результаты и их обсуждение}

\section{1. Характеризация полученного оксида вольфрама}

На рис. 1 приведены данные РФА образцов нанокристаллического оксида вольфрама.

Установлено, что образцы, приготовленные при разных температурах, представляют собой однофазный кристаллический продукт $\mathrm{WO}_{3}$, соответствующий карточке № 32-1395 в базе данных порошковых дифрактограмм PDF2.

Из данных рентгенофазового анализа (табл. 1) можно сделать следующие выводы. При повышении температуры обработки паравольфрамата аммония размер областей когерентного рассеяния (ОКР) оксида вольфрама возрастает, аналогично возрастает размер частиц, вычисленный по данным БЭТ, при этом одновременно уменьшается удельная площадь поверхности, а размер агрегатов соответственно возрастает.

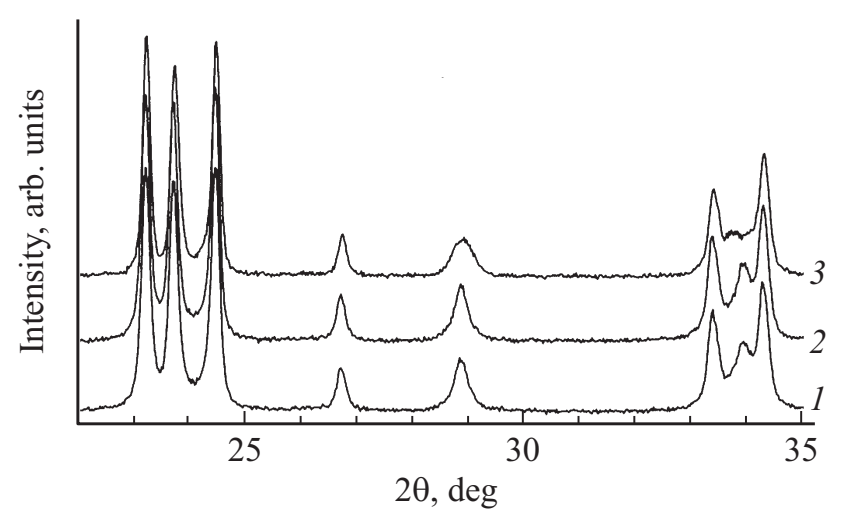

Рис. 1. Рентгенограммы порошков оксида вольфрама: $1-\mathrm{WO}_{3}(600), 2-\mathrm{WO}_{3}(700), 3-\mathrm{WO}_{3}(800)$. 


\section{2. Диэлектрическая проницаемость и диэлектрические потери}

Диэлектрическая проницаемость $(\varepsilon)$ является комплексной величиной,

$$
\varepsilon=\varepsilon^{\prime}+i \varepsilon^{\prime \prime},
$$

причем действительная, $\varepsilon^{\prime}$, и мнимая, $\varepsilon^{\prime \prime}$, части зависят от частоты $(v)$ и времени релаксации $(\tau)$ в соответствии с формулой Дебая, а отношение $\varepsilon^{\prime \prime} / \varepsilon^{\prime}=\operatorname{tg} \delta$ определяет диэлектрические потери в среде. Исследование частотных и температурных зависимостей $\varepsilon^{\prime}, \varepsilon^{\prime \prime}$ и $\operatorname{tg} \delta$ является одним из важных физических методов изучения свойств диэлектриков, позволяющих дифференцировать различные виды поляризации в твердом теле (электронная, ионная, дипольная, поляризация, обусловленная пространственными зарядами, и др.), а также получать качественную и количественную информацию о природе релаксационных и резонаторных центров в твердом теле (см., например, [19]).

На рис. 2 и 3 показаны соответственно частотные зависимости $\varepsilon^{\prime}$ и $\varepsilon^{\prime \prime}$ при различных температурах для образца $\mathrm{WO}_{3}(600)$ с размером наночастиц $d=110$ нм. Для образцов $\mathrm{WO}_{3}$ с размером наночастиц 150 и 200 нм зависимости имеют аналогичный вид.

Из данных рис. 2,3 видно, что в обоих случаях частотные зависимости могут быть описаны выражениями типа $\lg \left[\varepsilon^{\prime}\left(\varepsilon^{\prime \prime}\right)\right] \propto \lg (v)$. Имеются две ярко выраженные области с различным наклоном: относительно сильная зависимость от частоты в области низких частот и слабая зависимость от частоты в диапазоне высоких частот, при этом величины $\varepsilon^{\prime}$ и $\varepsilon^{\prime \prime}$ в высокочастотной области асимптотически стремятся к постоянной величине. Точка перегиба кривых закономерно смещается с увеличением температуры, при этом для зависимостей $\lg \left(\varepsilon^{\prime}\right) \propto \lg (v)$ частота меняется в диапазоне от 2 до 80 Гц; для зависимостей $\lg \left(\varepsilon^{\prime \prime}\right) \propto \lg (v)$ точка перегиба находится значительно выше - в области от 400 до 5000 Гц. Обращают на себя внимание достаточно большие значения величин $\varepsilon^{\prime}$ и $\varepsilon^{\prime \prime}$ в низкочастотной области, вероятно, связанных с поляризацией пространственного заряда на границах наночастиц оксида вольфрама, что обусловлено размером частиц и их совершенством. Измерения показали, что для образца $\mathrm{WO}_{3}(600)$ с наиболее низкой температурой отжига и соответственно наименьшим размером частиц, диэлектрическая проницаемость максимальная, с повышением же размера частиц диэлектрическая проницаемость снижается.

Поскольку диэлектрические потери - это одно из проявлений общего явления самопроизвольного перехода энергии упорядоченного движения в энергию хаотичного теплового движения, мощность потерь энергии электрического поля пропорциональна тангенсу угла диэлектрических потерь $(\operatorname{tg} \delta)$, а дисперсия $\operatorname{tg} \delta$ определяется дисперсией диэлектрической проницаемости и при релаксационном характере дисперсии максимум

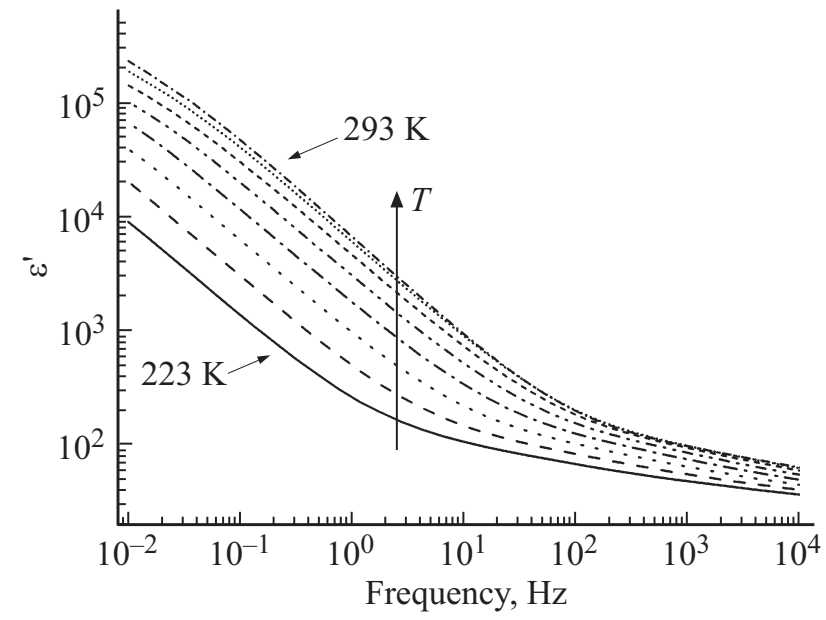

Рис. 2. Зависимость $\varepsilon^{\prime}$ от частоты при разных температурах для образца $\mathrm{WO}_{3}(600)$. Шаг изменения температуры $10 \mathrm{~K}$.

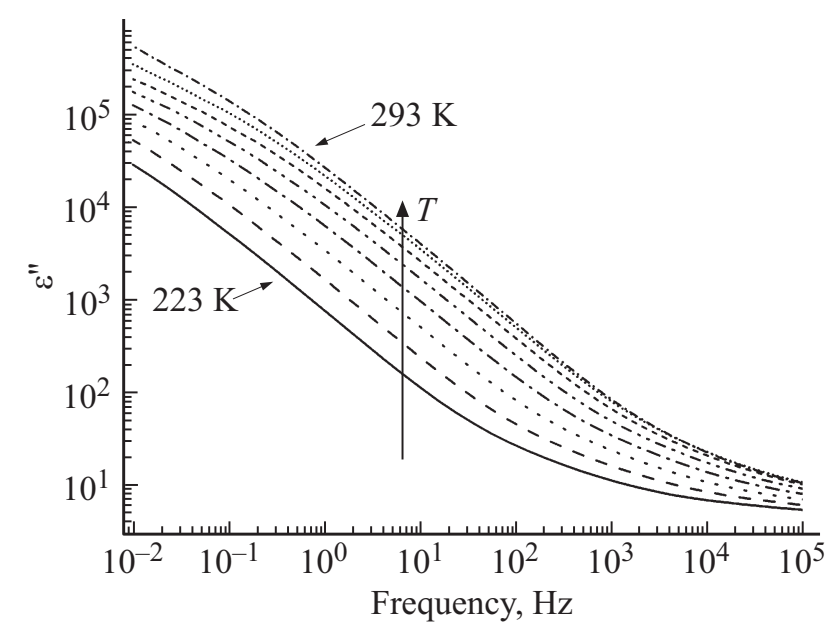

Рис. 3. Зависимость $\varepsilon^{\prime \prime}$ от частоты при разных температурах для образца $\mathrm{WO}_{3}(600)$. Шаг изменения температуры $10 \mathrm{~K}$.

диэлектрических потерь соответствует частоте

$$
v_{\max }=\frac{\sqrt{\varepsilon_{0}^{\prime} / \varepsilon_{\infty}^{\prime}}}{2 \pi \tau},
$$

где $\tau-$ время релаксации процессов поляризации, $\varepsilon_{0}^{\prime}$ - значение $\varepsilon^{\prime}$ при $2 \pi \nu \tau \ll 1, \varepsilon_{\infty}^{\prime}-$ значение $\varepsilon^{\prime}$ при $2 \pi v \tau \gg 1$, согласно [20]. На рис. 4 представлены кривые дисперсии для образца $\mathrm{WO}_{3}(600)$ с размером наночастиц 110 нм; для остальных образцов с размером наночастиц 150 и 200 нм зависимости имеют аналогичный вид.

Как видно из рис. 4 , дисперсии представляют кривые с максимумом, при этом с ростом температуры максимум закономерно смещается в высокочастотную область от 0.1 до $\sim 10$ Гц. Рост величины $\operatorname{tg} \delta$ с температурой в низкочастотной области обусловлен увеличением термически активированных свободных носителей и ростом потерь на токи сквозной проводимости. Подобное поведение характерно для диэлектрических пленок [21]. 


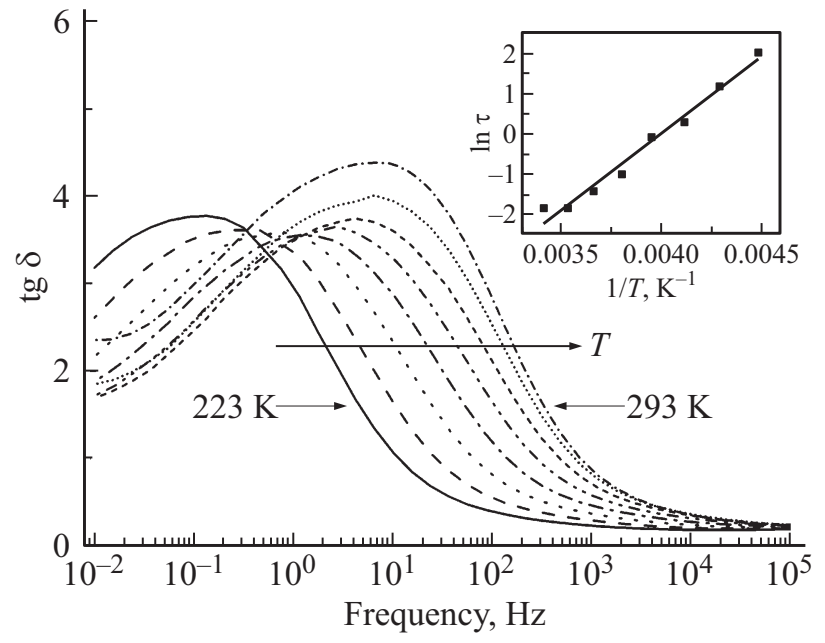

Рис. 4. Частотные зависимости $\operatorname{tg} \delta$ при различных температуpax для $\mathrm{WO}_{3}(600)$ с размером частиц 110 нм. Шаг изменения температуры $10 \mathrm{~K}$. На вставке - аррениусовская зависимость времени релаксации $\tau$.

Были рассчитаны времена $\tau$, характеризующие установление ориентационной поляризации полярных частиц. По температурной зависимости $\tau=f(1 / T)$, представленной на вставке, рассчитана величина потенциального барьера $E_{\text {rel }}$, разделяющего состояния с различными ориентациями, в предположении, что

$$
\tau=\tau_{0} \exp \left(E_{\text {rel }} / k_{\mathrm{B}} T\right)
$$

где $\tau_{0}-$ постоянная времени релаксации при $T \rightarrow \infty$, $k_{\mathrm{B}}-$ постоянная Больцмана.

Согласно результатам наших экспериментов, время релаксации лежит в пределах от 10 до $10^{3}$ с в зависимости от температурного диапазона, что существенно больше, чем времена ориентации электрических диполей, лежащие в диапазоне $10^{-6}-10^{-8}$ с, согласно [22]. Мы предполагаем, что наблюдаемая релаксационная дисперсия обусловлена дипольной ориентацией крупных частиц, например, комплексов с участием кислородных вакансий или неоднородностей нанокристаллического оксида вольфрама, которые связаны с размерами наночастиц.

В табл. 2 представлены величины потенциального барьера в зависимости от размера наночастиц, из которой видно, что с ростом размера частиц энергия потенциального барьера увеличивается. Это обусловлено

Таблица 2. Рассчитанные значения энергии активаци для разных процессов

\begin{tabular}{c|c|c|c}
\hline $\begin{array}{c}\text { Образец/ } \\
\text { размер нано- } \\
\text { частиц, нм }\end{array}$ & $\mathrm{WO}_{3}(600) / 110$ & $\mathrm{WO}_{3}(700) / 150$ & $\mathrm{WO}_{3}(800) / 220$ \\
\hline$E_{\text {rel, эВ }}$ & $0.33 \pm 0.02$ & $0.39 \pm 0.02$ & $0.44 \pm 0.03$ \\
$E_{\sigma}$, эВ & $0.32 \pm 0.01$ & $0.30 \pm 0.02$ & $0.29 \pm 0.01$
\end{tabular}

тем, что ориентация частиц большего размера будет происходить с бо́льшими энергетическими затратами. По абсолютной величине вычисленные значения достаточно хорошо коррелируют с аналогичным значением $E_{a}=0.301$ эВ, которое было получено в работе [16] для пленок $\mathrm{WO}_{3}$.

\section{3. Электропроводность нанокристаллического $\mathrm{WO}_{3}$}

На рис. 5 показаны частотные зависимости проводимости при различных температурах в диапазоне $223-293 \mathrm{~K}$ для $\mathrm{WO}_{3}(600)$ с размером наночастиц 110 нм; для остальных образцов зависимости имеют аналогичный вид. Во всех случаях наблюдается возрастание проводимсости на переменном токе с частотой $\sigma_{a c}(v)$, что обусловлено запаздыванием медленных механизмов поляризации. Общим для всех исследованных образцов является степенной характер зависимости,

$$
\sigma_{a c}(v) \propto v^{s},
$$

где $s$ - показатель степени, характеризующий наклон линейной части зависимости и лежащий в диапазоне 0.7-1.0 для широкого круга материалов с различными составом и структурой, согласно работам [23,24]. Такая зависимость свойственна многим механизмам перемещения заряженных частиц (комплексов) в электрическом поле, общим для которых является их перемещение в ограниченном пространстве. Значение показателя $s$ позволяет сделать предположения о механизме проводимости.

Аппроксимация экспериментальных кривых в области $10^{4}-10^{6}$ Гц функцией, соответствующей уравнению (4), показала, что значение показателя степени варьируется в диапазоне $s=(0.83-0.90) \pm 0.01$ в зависимости от

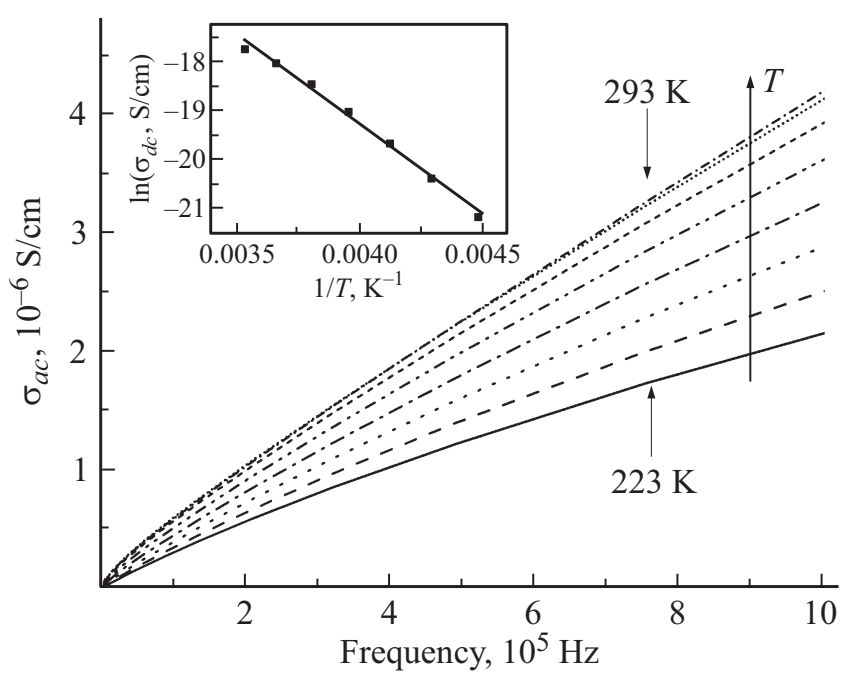

Рис. 5. Зависимости проводимости на переменном токе для $\mathrm{WO}_{3}(600)$ с размером наночастиц 110 нм при различных температурах. Шаг изменения температуры $10 \mathrm{~K}$. На вставке - аррениусовская зависимость проводимости на постоянном токе. 
температуры: с ростом температуры параметр $s$ увеличивается. Наблюдается рост проводимости $\sigma_{a c}$ с температурой и частотой, причем дисперсия ярко выражена в области высоких частот, в области же низких частот зависимость менее выраженная. Частотная зависимость и величина показателя $s$ могут свидетельствовать в пользу механизма прыжковой проводимости по локализованным состояниям, ограниченным потенциальными барьерами и связанным с дефектами структуры, например с кислородными вакансиями.

Таким образом, общая тенденция для частотных зависимостей, представленных на рис. 2, 3 и 5, может быть объяснена следующим. Тепловые движения заряженных частиц, локализация которых определяется наличием потенциальных барьеров, во внешнем электрическом поле приводят как к проводимости, так и к поляризации. В области низких частот $(v \rightarrow 0)$ преобладают процессы поляризации, потому что пространственное движение заряженных частиц в электрическом поле низкой частоты ограничено потенциальными барьерами, дефектами структуры и границами раздела наночастиц, которые препятствуют полному переносу электрических зарядов в структуре. По мере повышения частоты электрического поля заряженные частицы не успевают за время четверти периода приложенного напряжения достигнуть мест своей локализации и, непрерывно следуя за изменением электрического поля, дают вклад уже в проводимость. При этом вклад от их движения в поляризацию снижается, вследствие чего наблюдается дисперсия диэлектрической проницаемости. Большое различие в величине потенциальных барьеров и в длине „свободного пробега“ заряженных частиц объясняет непрерывный рост проводимости и соответствующее снижение $\varepsilon$, наблюдаемые в эксперименте в широком частотном интервале.

В предположении, что при $v \rightarrow 0$ проводимость является проводимостью на постоянном токе, $\sigma_{d c}$, мы использовали экстраполированные значения $\sigma_{a c}$ к $v=0$ в качестве $\sigma_{d c}$ и по аррениусовской зависимости

$$
\sigma_{d c}=A \exp \left(-E_{\sigma} / k_{\mathrm{B}} T\right)
$$

где $A$ - предэкспоненциальный множитель, мы определили энергию активации проводимости $E_{\sigma}$. Рассчитанные значения приведены в табл. 2.

Как видно из данных табл. 2, значения $E_{\sigma}$ коррелируют по абсолютной величине с данными для $E_{\mathrm{rel}}$, однако тенденции различаются: с ростом размера наночастиц $E_{\sigma}$ незначительно уменьшается, в то время как $E_{\mathrm{rel}}$ закономерно растет. Близость абсолютных значений $E_{\mathrm{rel}}$ и $E_{\sigma}$ может свидетельствовать о том, что в области низких частот и пониженных температур для нанокристаллического оксида вольфрама термически активируемые релаксационные процессы и процессы переноса носителей заряда по локализованным состояниям обусловлены одной причиной - например, наличием дефектов типа кислородных вакансий в материале. Следует заметить, что рассчитанные нами значения энергии активации проводимости значительно отличаются от результатов, полученных в [18] для пленок аморфного $\mathrm{WO}_{3}$, которые дают значение, равное $E_{d c}=(0.63 \pm 0.03)$ эВ, что говорит о влиянии фазового состава и способа приготовления образцов на электрические характеристики оксида вольфрама.

\section{4. Заключение}

Исследованы диэлектрические свойства нанокристаллического оксида вольфрама $\mathrm{WO}_{3}$ в интервале температур $223-293 \mathrm{~K}$ и в диапазоне частот $10^{-2}-10^{6}$ Гц. Образцы были приготовлены термической обработкой паравольфрамата аммония при температурах 600, 700 и $800^{\circ} \mathrm{C}$, что позволило получить порошки с размером частиц 110,150 и $200 \mathrm{Hм}$ соответственно. Установлено, что для всех образцов частотные зависимости проводимости являются возрастающими с увеличением частоты, в то время как поляризационные характеристики $\varepsilon^{\prime}(v)$ и $\varepsilon^{\prime \prime}(v)$ - падающие. Найдено, что частотные зависимости проводимости описываются функцией вида $v^{s}$ с показателем степени, находящимся в диапазоне $(0.83-0.90) \pm 0.01$, что характерно для „прыжкового“ механизма перемещения заряженных частиц (комплексов) между локализованными состояниями под действием электрического поля. Показано, что поляризационные процессы и процессы проводимости являются термически активируемыми, найденные значения энергии активации лежат в диапазоне от 0.3 до 0.4 эВ в зависимости от размера наночастиц.

Исследование выполнено при поддержке гранта Российского научного фонда (проект №16-13-10399).

\section{Список литературы}

[1] G. Granqvist. Handbook of Electrochromic Materials (Amsterdam, Elsevier, 1995).

[2] B. Urasinska-Wojcik, T.A. Vincent, M.F. Chowdhury, J.W. Gardner. Sensors Actuators B: Chemical, 239, 1051 (2017).

[3] L. Wang, A. Teleki, S.E. Pratsinis, P.I. Gouma. Chem. Mater., 20, 4794 (2008).

[4] S.S. Kalaga, S.S. Mali, D.S. Dalavi, A.I. Inamdar, H. Im, P.S. Patil. Synth. Metals, 161, 1105 (2011).

[5] S.E.M. Svensson, C.G. Granqvist. Thin Sol. Films, 126, 31 (1985).

[6] B. Gavanier, F.M. Miehalak, J.R. Owen. Ionics, 3, 265 (1997).

[7] G. Leftheriotis, G. Syrrokostas, P. Yianoulis. Solar Energy Mater. Solar Cells, 94, 2304 (2010).

[8] A. Pawlicka. Recent Patents on Nanotechnology, 3, 177 (2009).

[9] S. Long, H. Zhou, S. Bao, Y. Xin, X. Cao, P. Jin. RCS Advances, 6, 106435 (2016).

[10] Ch. Ma, Ch. Zhou, Zh. Zhang, B. Wang, L. Wei. Mater. Sci. Forum, 445\&446, 141 (2004).

[11] E.K.H. Salje. Eur. J. Solid State Inorg. Chem., 31, 651 (1994).

[12] A. Aird, M.C. Domeneghetti, F. Mazzi, V. Tazzoli, E.K.H. Salje. J. Phys.: Condens. Matter, 10, L569 (1998). 
[13] Sh. Sawada. J. Phys. Soc. Japan, 11 (12), 1237 (1956).

[14] L. Wang, A. Teleki, S.E. Pratsinis, P.I. Gouma. Chem. Mater., 20, 4794 (2008).

[15] P.M. Woodward, A.W. Sleight, T. Vogt. J. Solid State Chem., 131 (1), 9 (1997).

[16] A. Elshafie, H.H. Afify, A. Abdel-All. Phys. Status Solidi, 174, 301 (1999).

[17] M.M. El-Nahass, H.A.M. Ali, M. Saadeldin, M. Zaghllol. Physica B, 407, 4453 (2012).

[18] M.G. Hutchins, O. Abu-Alkhair, M.M. El-Nahass, K. AbdelHady. J. Non-Cryst. Sol., 353, 4137 (2007).

[19] Г. Фрелих. Теория диэлектриков (М., Мир, 1960).

[20] П.Т. Орешкин. Физика полупроводников и диэлектриков (М., Высш. шк., 1977).

[21] B. Jagan Mohan Reddy, G. Paran Jyothi, M.V. Ramana Reddy, M.N. Chary, Narasimha Reddy. Phys. Status Solidi, 137, 241 (1993).

[22] Н. Ашкрофт, Н. Мермин. Физика твердого тела (М., Мир, 1979) т. 2.

[23] А. Фельц. Аморбные и стеклообразные неорганические твердые тела (М., Мир, 1986).

[24] Ю.М. Поплавко, Л.П. Переверзева, И.П. Раевский. Физика активных диэлектриков, под ред. В.П. Сахненко (Ростов н/Д, Изд-во ЮФУ, 2009).

Редактор Л.В. Шаронова

\section{Dielectric properties of nanocrystalline tungsten oxide in the temperature range 223-293 K}

S.A. Kozyukhin 1,3, S.A. Bedin' ${ }^{2}$, P.G. Rudakovskaya ${ }^{1}$, O.S. Ivanova ${ }^{1}$, V.K. Ivanov ${ }^{1,4}$

${ }^{1}$ Kurnakov Institute of General and Inorganic Chemistry, Russian Academy of Sciences, 119991 Moscow, Russia

${ }^{2}$ Moscow Pedagogical State University,

119991 Moscow, Russia

${ }^{3}$ National Research Tomsk State University,

(Faculty of Chemistry),

634050 Tomsk, Russia

${ }^{4}$ Moscow University of Technology,

119454 Moscow, Russia

Abstract The dielectric properties of nanocrystalline tungsten oxide in the temperature range $223-293 \mathrm{~K}$ and in the frequency range $v=10^{-2}-10^{6} \mathrm{~Hz}$ were studied. $\mathrm{WO}_{3}$ powders with particle sizes of 110,150 and $200 \mathrm{~nm}$ were prepared by thermal treatment of ammonium paratungstate at various temperatures. It was established that the frequency dependences of the conductivity is increasing with increasing frequency for all samples, while the polarization characteristics $\varepsilon^{\prime}(v)$ and $\varepsilon^{\prime \prime}(v)$ are decreasing. It was found that the frequency dependences of the conductivity may be described by a function of $v^{s}$ with an exponent in the range $(0.83-0.90) \pm 0.01$, which is characteristic for the hopping conduction mechanism of charged particles (complexes) over localized states limited by potential barriers and structural defects. 